Molecular basis of proton uptake in single and double mutants of cytochrome $c$ oxidase

This content has been downloaded from IOPscience. Please scroll down to see the full text.

2011 J. Phys.: Condens. Matter 23234102

(http://iopscience.iop.org/0953-8984/23/23/234102)

View the table of contents for this issue, or go to the journal homepage for more

Download details:

IP Address: 149.157.61.163

This content was downloaded on 21/07/2016 at $11: 58$

Please note that terms and conditions apply. 


\title{
Molecular basis of proton uptake in single and double mutants of cytochrome $c$ oxidase
}

\author{
Rowan M Henry $^{1,2}$, David Caplan ${ }^{1,2}$, Elisa Fadda ${ }^{3}$ and \\ Régis Pomès ${ }^{1,2,4}$ \\ ${ }^{1}$ Molecular Structure and Function, Hospital for Sick Children, Toronto, ON, M5G 1X8, \\ Canada \\ ${ }^{2}$ Department of Biochemistry, University of Toronto, Toronto, ON, M5S 1A8, Canada \\ ${ }^{3}$ Department of Chemistry, University of Galway, Ireland \\ E-mail: pomes@sickkids.ca
}

Received 10 December 2010, in final form 15 March 2011

Published 25 May 2011

Online at stacks.iop.org/JPhysCM/23/234102

\begin{abstract}
Cytochrome $c$ oxidase, the terminal enzyme of the respiratory chain, utilizes the reduction of dioxygen into water to pump protons across the mitochondrial inner membrane. The principal pathway of proton uptake into the enzyme, the $\mathrm{D}$ channel, is a $2.5 \mathrm{~nm}$ long channel-like cavity named after a conserved, negatively charged aspartic acid (D) residue thought to help recruiting protons to its entrance (D132 in the first subunit of the $S$. sphaeroides enzyme). The single-point mutation of D132 to asparagine $(\mathrm{N})$, a neutral residue, abolishes enzyme activity. Conversely, replacing conserved N139, one-third into the D channel, by D, induces a decoupled phenotype, whereby oxygen reduction proceeds but not proton pumping. Intriguingly, the double mutant D132N/N139D, which conserves the charge of the D channel, restores the wild-type phenotype. We use molecular dynamics simulations and electrostatic calculations to examine the structural and physical basis for the coupling of proton pumping and oxygen chemistry in single and double N139D mutants. The potential of mean force for the conformational isomerization of N139 and N139D side chains reveals the presence of three rotamers, one of which faces the channel entrance. This out-facing conformer is metastable in the wild-type and in the N139D single mutant, but predominant in the double mutant thanks to the loss of electrostatic repulsion with the carboxylate group of D132. The effects of mutations and conformational isomerization on the $p \mathrm{Ka}$ of $\mathrm{E} 286$, an essential proton-shuttling residue located at the top of the D channel, are shown to be consistent with the electrostatic control of proton pumping proposed recently (Fadda et al 2008 Biochim. Biophys. Acta 1777 277-84). Taken together, these results suggest that preserving the spatial distribution of charges at the entrance of the D channel is necessary to guarantee both the uptake and the relay of protons to the active site of the enzyme. These findings highlight the interplay of long-range electrostatic forces and local structural fluctuations in the control of proton movement and provide a physical explanation for the restoration of proton pumping activity in the double mutant.

(Some figures in this article are in colour only in the electronic version)
\end{abstract}

\section{Introduction}

Cytochrome $c$ oxidase, an intrinsic membrane protein, is the fourth enzyme complex in the respiratory chain. Its role is

4 Address for correspondence: Molecular Structure and Function, Hospital for Sick Children, 555 University Avenue, Toronto, ON, M5G 1X8, Canada. to convert dioxygen to water and harness the energy liberated from this redox reaction to pump protons across the membrane against an electrochemical gradient (for a review see [1-3]). The resulting proton-motive force is utilized by ATP synthase to drive the synthesis of ATP. The redox reaction occurs in a stepwise manner and takes place in the binuclear centre 


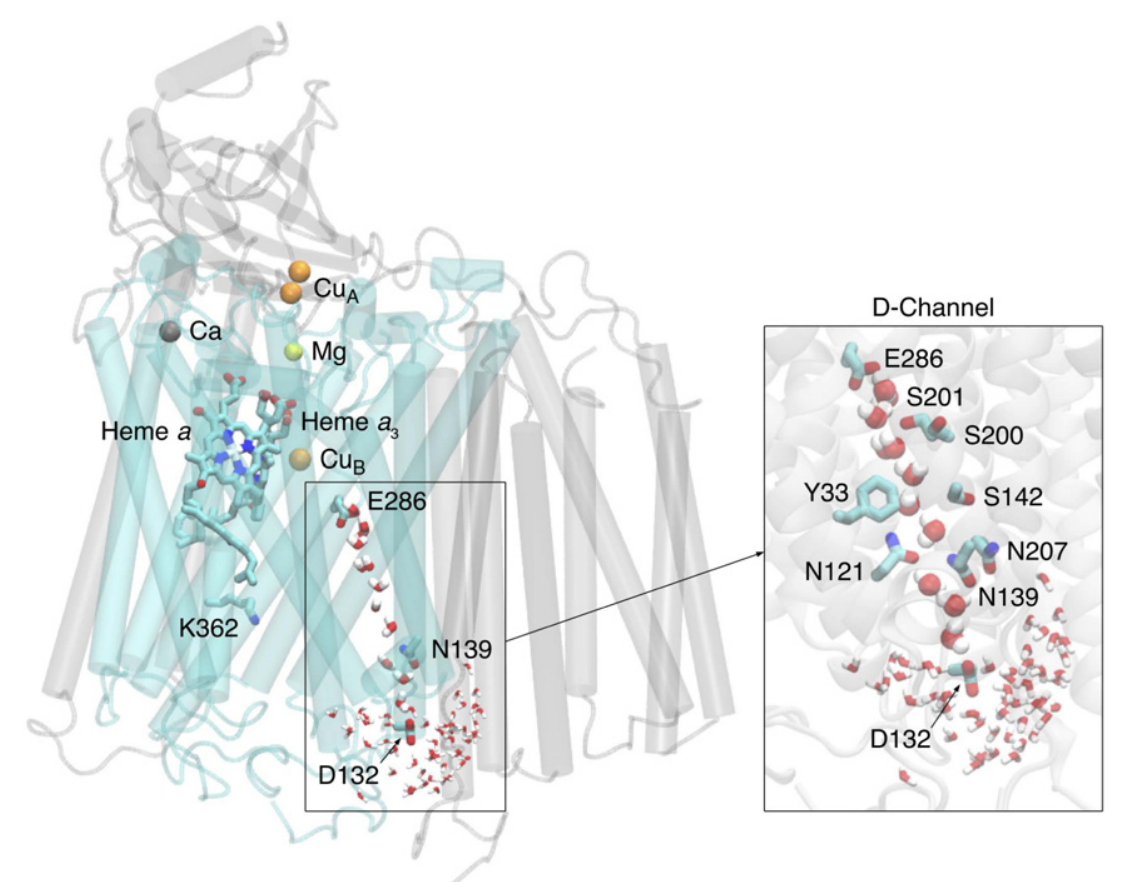

Figure 1. Cytochrome $c$ oxidase of $R$. sphaeroides. Subunit I, which contains the D channel (inset) and the binuclear centre, is highlighted in blue. Highlighted are heme a, heme a3, $\mathrm{Cu}_{\mathrm{A}}, \mathrm{Cu}_{\mathrm{B}}$ and key residues of the $\mathrm{D}$ channel, which extends from D132 to E286. This conformation is taken from one of our simulations with N139 in the open state and a chain of 12 water molecules in the D channel. Also pictured is the cap of 57 water molecules at the entrance of the $\mathrm{D}$ channel used to represent bulk solution.

(BNC), which includes a copper centre $\left(\mathrm{Cu}_{\mathrm{B}}\right)$ and a high-spin Fe-heme (heme a3) (see figure 1). Throughout the catalytic cycle, four electrons are obtained from the reduction of a bimetallic $\mathrm{Cu}$ centre, $\mathrm{Cu}_{\mathrm{A}}$, by cytochrome $c$. The electrons are then transferred from $\mathrm{Cu}_{\mathrm{A}}$ to the low-spin heme complex heme a. The reaction cycle utilizes eight protons, four of which are consumed in the redox reaction and four of which are translocated to the exit pathway towards the cytoplasmic side of the membrane via the proton loading site (PLS). All eight protons are taken up from the matrix side of the mitochondrial membrane and transported to the active site through two cavities in the enzyme, the D channel and the $\mathrm{K}$ channel, which are named after highly conserved Asp132 and Lys362 residues, respectively (numbering is from subunit I of $R$. sphaeroides $\mathrm{Cc} \mathrm{O}$ unless otherwise noted) [1-3].

The majority of the protons used in the reaction cycle are taken up from the D channel $[4,5]$. The D channel is located in subunit I and extends approximately $25 \AA$ from residue D132 at the entrance to residue E286, itself approximately $12 \AA$ from the binuclear centre (figure 1). The negatively charged group of residue D132 has been proposed to act as a 'proton antenna', recruiting protons from the matrix side to be transferred through the D channel [6]. Once inside the $\mathrm{D}$ channel, an excess proton is thought to be relayed throughout the cavity by a Grotthuss-like mechanism [7, 8] involving successive exchanges of hydrogen nuclei in a chain of water molecules forming an extended hydrogen-bonded network [9]. Such a mechanism is supported by the presence of water providing a pathway for the relay of protons in the D channel $[10,11]$ and by simulations of proton movement in the hydrated cavity forming the upper half of the D channel [12].
At the 'top' of the D channel, residue E286 plays an essential role in the catalytic activity by shuttling protons from the D channel to both the BNC and the PLS [13-16]. Point mutations of some of the residues lining the $\mathrm{D}$ channel have been observed to significantly affect the activity of the enzyme and highlight the importance of the D channel to the mechanism of redox-coupled proton pumping (see table 1) [4, 6, 17-26]. In particular, single-point mutations of residues N139 and N207 to an aspartic acid residue produce a decoupled phenotype, whereby the protein maintains wild-type turnover (i.e. redox activity) while completely abolishing proton pumping [20, 23]. Moreover, the single-point mutation G204D eliminates all protein activity [22]. Similarly, neutralization of the presumed proton antenna in the single-point mutation D132N also results in an inactive enzyme [6]. Most intriguing, combining the D132N mutation with the N139D mutant (D132N/N139D) restores wild-type activity to the formerly decoupled or inactive enzyme [17]. These findings indicate that, despite its distant proximity from the BNC and its presumably passive role as a proton conduit in the uptake and relay of protons, the $\mathrm{D}$ channel plays a vital role in the catalytic mechanism of the enzyme. For this reason, uncovering the molecular basis of proton uptake and relay via the $\mathrm{D}$ channel will lead to a better understanding of proton pumping.

Numerous models of the catalytic mechanism of $\mathrm{CcO}$ have been proposed, all of which identify five distinct catalytic states [27-31]. However, the mechanism which couples redox chemistry and proton pumping remains unclear. Recently, we proposed a model for the catalytic cycle of $\mathrm{CcO}$ which rests on the control of vectorial proton transfer by long-range electrostatic interactions in a recurring sequence of electron 
Table 1. Summary of oxygen redox activity and proton pumping in the wild-type and in D-channel site-directed mutants of cytochrome $c$ oxidase.

\begin{tabular}{|c|c|c|c|c|}
\hline Organism & Mutation & Activity (\% of WT) & Pumping & Reference \\
\hline R. sphaeroides & WT & 100 & Yes & - \\
\hline R. sphaeroides & $\mathrm{Y} 33 \mathrm{H}$ & 40 & No & [44] \\
\hline R. sphaeroides & N121A & 110 & Reduced & [45] \\
\hline R. sphaeroides & $\mathrm{N} 121 \mathrm{~T}$ & 100 & No & [45] \\
\hline R. sphaeroides & N121D & 70 & No & [45] \\
\hline R. sphaeroides & D132A & 5 & No & {$[6,46]$} \\
\hline R. sphaeroides & D132N & 5 & No & [6] \\
\hline E. coli & $\mathrm{D} 135 \mathrm{~N}(\mathrm{D} 132 \mathrm{~N})^{\mathrm{a}}$ & 45 & Reduced & [26] \\
\hline R. sphaeroides & N139D & $150-300$ & No & {$[20,47]$} \\
\hline P. denitrificans & N131D (N139D) & $100-115$ & No & {$[25]$} \\
\hline E. coli & N142D (N139D) & 50 & Reduced & [26] \\
\hline R. sphaeroides & N139A & 40 & No & [45] \\
\hline P. denitrificans & N131A (N139A) & 11 & No & [48] \\
\hline R. sphaeroides & N139C & 110 & No & {$[45]$} \\
\hline P. denitrificans & N131C (N139C) & 86 & No & [48] \\
\hline R. sphaeroides & N139Q & 60 & Reduced & {$[45]$} \\
\hline P. denitrificans & N131Q (N139Q) & 52 & Reduced & [48] \\
\hline E. coli & N142V (N139V) & 109 & Yes & [26] \\
\hline R. sphaeroides & N139T & 40 & No & [49] \\
\hline R. sphaeroides & N139E & 110 & No & {$[45]$} \\
\hline P. denitrificans & N131E (N139E) & 78 & No & [48] \\
\hline R. sphaeroides & N139L & 7 & No & {$[45]$} \\
\hline R. sphaeroides & N139S & 90 & No & [45] \\
\hline P. denitrificans & N131V (N139V) & $<10$ & No & {$[25]$} \\
\hline E. coli & N142V (N139V) & 22 & Reduced & [26] \\
\hline R. sphaeroides & S142A & 80 & Reduced & [45] \\
\hline P. denitrificans & S134A (S142A) & 96 & Yes & {$[25]$} \\
\hline R. sphaeroides & S142D & 2 & No & {$[45]$} \\
\hline R. sphaeroides & S200I & 83 & Yes & {$[50]$} \\
\hline R. sphaeroides & G204D & 2 & No & [22] \\
\hline R. sphaeroides & N207D & 100 & No & [23] \\
\hline P. denitrificans & N199D (N207D) & 50 & No & {$[25]$} \\
\hline R. sphaeroides & N207A & 90 & Reduced & [45] \\
\hline R. sphaeroides & N207T & 90 & Reduced & {$[45]$} \\
\hline P. denitrificans & N113V/N131D (N121V/N139D) & 75 & No & [25] \\
\hline R. sphaeroides & D132N/N139D & 20 & Yes & [17] \\
\hline E. coli & D135N/N142D (D132N/N139D) & 33 & Yes & [26] \\
\hline R. sphaeroides & D132N/N139T & 90 & No & {$[45]$} \\
\hline R. sphaeroides & D132N/S200I & 3 & No & [50] \\
\hline R. sphaeroides & D132N/S200V/S201V & 7 & No & {$[50]$} \\
\hline R. sphaeroides & D132N/S200V/S201Y & 7 & No & {$[50]$} \\
\hline R. sphaeroides & S142A/N207A & 80 & Reduced & [45] \\
\hline R. sphaeroides & S200V/S201V & 37 & Reduced & {$[50]$} \\
\hline R. sphaeroides & S200V/S201Y & 11 & No & {$[50]$} \\
\hline
\end{tabular}

and proton transfer steps occurring in the active site [32]. In this model, E286 plays a vital role by relaying most of the chemical and pumped protons to the BNC and the PLS, respectively. This role requires that its $p \mathrm{~K}_{\mathrm{a}}$ or proton affinity be at once high enough to guarantee proton uptake from the $\mathrm{D}$ channel and low enough to relay chemical protons on to the BNC and vectorial protons on to the PLS. To evaluate our model, the $p \mathrm{~K}_{\mathrm{a}}$ of E286 was calculated to gauge its ability to deliver chemical and vectorial protons throughout the catalytic cycle, successively in the wild-type and in the N139D, N207D and G204 mutants. This study ascribed the mutant phenotypes to long-range electrostatic forces. Results showed that the introduction of an anionic group into the $\mathrm{D}$ channel induces an increase in the $p \mathrm{~K}_{\mathrm{a}}$ of E286 and suggested that the decoupled (N139D, N207D) and inactive (G204D) phenotypes stem from compromised delivery of vectorial and chemical protons due to this elevated $p \mathrm{~K}_{\mathrm{a}}$. In this process, the magnitude of the $\mathrm{pKa}$ shift is modulated by the proximity of the anionic charge to the carboxylic group of E286. In the case of the decoupled mutants, the $p \mathrm{~K}_{\mathrm{a}}$ of E286 was raised significantly, which may still enable the delivery of chemical protons, but is too high to deliver pumped protons. In the inactive G204D mutant, whose side chain lies closer to E286 by $3 \AA$, the $p \mathrm{~K}_{\mathrm{a}}$ of E286 further increased by an additional unit relative to the wild-type, which is presumably too high to allow any proton delivery.

Absent from our previous study was an analysis of the effect of the D132N/N139D double mutant on the $p \mathrm{~K}_{\mathrm{a}}$ of E286. This double mutant is particularly intriguing since it is able at once to restore activity to a decoupled mutant, N139D, and to an inactive mutant, D132N (see table 1). 
Since we have proposed that the phenotype of the singlepoint mutant $\mathrm{N} 139 \mathrm{D}$ is due to the altered $p \mathrm{~K}_{\mathrm{a}}$ of $\mathrm{E} 286$, the phenotype of the double mutant puts our electrostatic model to the test. If the decoupling of proton pumping in N139D is due to the introduction of a negative charge in the D channel, why is the double mutant, which conserves the charge of residue N139D, functional? In another development, our recent comparative study of functional hydration in the wild-type enzyme and in two uncharged single-point mutants (respectively alanine and valine, N139A and N139V) revealed the existence of multiple rotameric states in the side chain of residue N139 [11]. Specifically, this side chain was found to predominantly adopt the 'closed' conformation seen in the crystallographic structures [10], whereby it interrupts the hydrogen-bonded chain of water molecules in the D channel, and to adopt a metastable 'open' conformation in which hydrogen-bond connectivity is established and water-mediated proton relay may proceed according to a Grotthuss mechanism. Interestingly, the occlusion of this conformational gate in the $\mathrm{N} 139 \mathrm{~V}$ mutant corresponds to an inactive enzyme, whereas the removal of the gate in N139A corresponds to a decoupled phenotype, suggesting that conformational gating of proton uptake by residue N139 is indeed required for the proper function of the enzyme [11].

The existence of a conformational gate modulating proton uptake in the wild-type enzyme suggests that multiple rotameric states may also exist for the N139D side chain. If so, these states must be accounted for in our electrostatic model of proton pumping. To this end, we now examine the physical basis for the phenotype of single and double mutants. We first present systematic free energy calculations to characterize the conformational isomerization of the side chain of residue 139 successively in the wild-type protein and in the N139D and D132N/N139D mutants. We then assess the effect of this conformational isomerization on the $p \mathrm{~K}_{\mathrm{a}}$ of E286 using continuum electrostatic calculations. The results reveal the existence of an additional conformation in which the side chain of residue 139 faces out, towards the entrance of the D channel. This 'out-facing' conformer predominates in the double mutant, eliminating the shift in the $p \mathrm{~K}_{\mathrm{a}}$ of E286 induced by long-range electrostatic repulsion with N139D while putatively restoring the proton antenna capability compromised in the D132N mutant. Together, these findings offer a simple explanation for the phenotype of the double mutant consistent with the electrostatic control of proton uptake and delivery. By restoring the charge distribution of the enzyme at the entrance of the D channel, the double mutant restores the proton pumping activity of the wild-type enzyme compromised in the two single-point mutants.

\section{Methods}

\subsection{Molecular system}

The initial conformation of the protein was obtained from the structure of $R$. sphaeroides cytochrome $c$ oxidase solved at $2.3 \AA$ resolution by x-ray crystallography (PDB ID code 1M56) [10]. Mutant structures were obtained by performing mutations to N139 and D132 in silico, followed by 2 ns of equilibration at $298 \mathrm{~K}$. All simulations were performed with 12 water molecules present in the D channel following our systematic study of hydration [11]. As in that previous study, a hemispherical 'cap' of water molecules was placed at the entrance of the D channel to model bulk solution on the matrix side of the protein. The 57 water molecules were restrained by a spherical boundary potential with a radius of $9 \AA$ centred at the channel opening and a force constant of $5 \mathrm{kcal} \mathrm{mol}^{-1} \AA^{-2}$. An axis connecting the $\mathrm{C}_{\alpha}$ atoms of D132 and E286 was used to define the $\mathrm{D}$ channel and was aligned with the $z$ axis. Residues with at least one heavy atom within $5 \AA$ from the D-channel axis as well as the D-channel water molecules and cap water molecules were allowed to move during the MD simulations. All remaining atoms in the system were held fixed.

The CHARMM force field, version 22 [33], was used to model the protein, and the TIP3P force field [34] was used to model water molecules. The charge distribution of the binuclear centre was calculated as described in Fadda et al [32]. The enzyme was simulated in the fully reduced $R$ state. Titratable residues were simulated at standard protonation states. In particular, E286 was modelled in its deprotonated state to mimic conditions favourable to proton uptake in the D channel [32].

\subsection{Molecular dynamics simulations}

The MD trajectories were generated using the program CHARMM, version 28 [35]. The Langevin equations of motion were propagated at $298 \mathrm{~K}$ with an integration step of $2 \mathrm{fs}$ and a friction coefficient of $5 \mathrm{ps}^{-1}$ applied to all heavy atoms. The SHAKE algorithm [36] was employed to fix all covalent bonds involving hydrogen atoms with a bond deviation tolerance of $1.0 \times 10^{-6}$. Non-bonded interactions were calculated with a force-based switching function acting between 14 and $16 \AA$. Trajectories and structures were viewed using visual molecular dynamics (VMD) [37].

\subsection{Free energy calculations}

The reversible free energy change or potential of mean force (PMF) for the rotation about the $\mathrm{C}_{\alpha}-\mathrm{C}_{\beta}$ torsion angle $\chi_{1}$ of residue 139 was calculated using umbrella sampling (US) [38] together with distributed replica sampling (DR) [39] to reduce systematic sampling errors [40, 41]. In the US scheme, the $\chi_{1}$ torsion of residue 139 was subjected to confinement by quadratic biasing potentials of the form $V_{i}\left(\chi_{1}\right)=\frac{1}{2} k_{i}\left(\chi_{1}-\chi_{1, i}\right)^{2}$, where $k_{i}$ is the restoring force constant and $\chi_{1, i}$ ranged from $0^{\circ}$ to $320^{\circ}$ in $10^{\circ}$ increments. DR is a generalized-ensemble algorithm designed to improve the efficiency of Boltzmann sampling by achieving a random walk in temperature or (as in the present case) in conformational space [39, 42]. Multiple replicas of a protein system differing in reaction coordinate (here, umbrella restraint $V_{i}$ centred at $\chi_{1, i}$ ) are simulated independently. Periodically, individual replicas are halted and a stochastic move replacing $V_{i}$ by adjacent umbrella $V_{i \pm 1}$ is attempted. Construction 


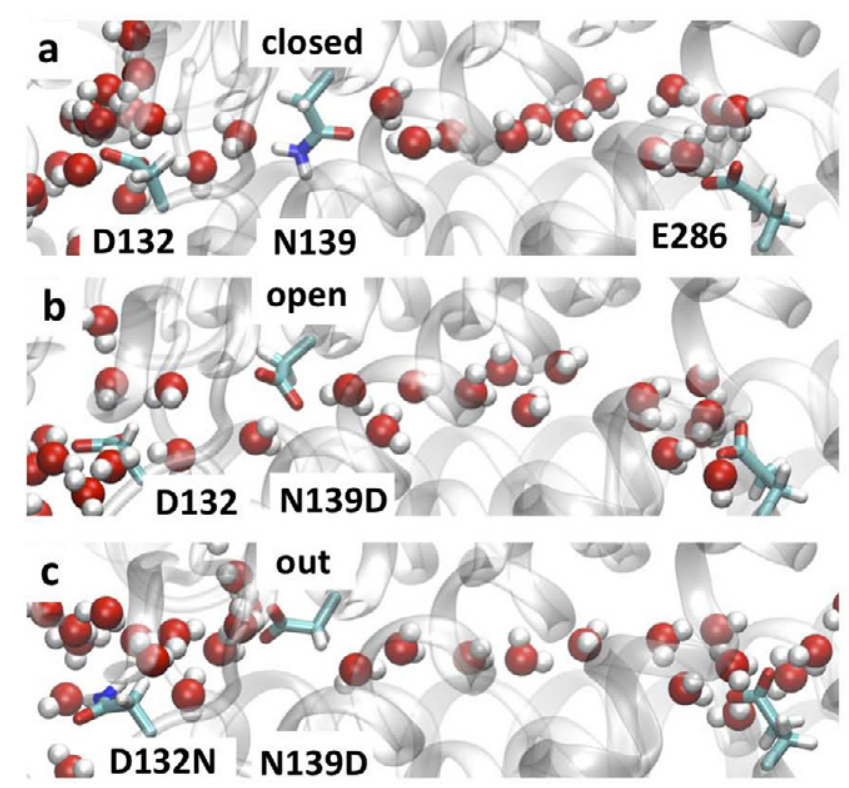

Figure 2. Representative snapshots of the D channel for three different conformations of residue 139: (a) closed (crystallographic) conformation of N139 in the wild-type enzyme; (b) open conformation of the N139D single-point mutant; and (c) out-facing conformation of N139D in the D132N/N139D double mutant. Water molecules present in or near the D channel are shown in spherical representation together with licorice representations of the side chains of residues 132,139 and 286 . The $\mathrm{C}_{\alpha}$ trace of the protein is depicted as ribbons.

of all starting structures began from equilibrated structures of residues $\mathrm{N} 139$ and N139D in their closed positions of $\chi_{1} \approx 195^{\circ}$ and $210^{\circ}$, respectively [11]. From these initial conformations, the remaining structures were generated by running successive $400 \mathrm{ps}$ simulations. Here, the final structure of a replica with $\chi_{1, i}=X$ was used as the starting structure for the replica with $\chi_{1, i}=X+10^{\circ}$ or $\chi_{1, i}=X-10^{\circ}$, as required. All replicas were then equilibrated for another $400 \mathrm{ps}$ with a force constant $k_{i}=0.02 \mathrm{kcal} / \mathrm{mol} / \mathrm{deg}^{2}$. Next, a series of $2 \mathrm{~ns}$ simulations of all replicas were performed while adjusting both the umbrella centres $\chi_{1, i}$ and force constants $k_{i}$ in an attempt to obtain a near $20 \%$ overlap between all adjacent umbrellas in the sampled $\chi_{1}$ space. This procedure resulted in small differences in the final umbrella positions and force constants for the wild-type, N139D, and D132N/N139D systems. Weight values (also called 'A values') required to achieve a random walk in the DR scheme were calculated adaptively as described before [40, 42] using $1 \mathrm{~ns}$ of simulation time per replica with no exchanges. All replicas were then run while allowing umbrella replacements every $2 \mathrm{ps.} \mathrm{The} \mathrm{total}$ sampling time was $631 \mathrm{~ns}, 772 \mathrm{~ns}$ and $834 \mathrm{~ns}$ for the wild-type, N139D and D132N/N139D systems, respectively. Throughout the simulation, weight values were adjusted based on the current calculated free energy surface. PMFs were generated using Alan Grossfield's implementation of WHAM [43]. Error was calculated using block averaging. A separate PMF was generated for each block of data and a global fit was used to align all the PMFs thus generated. The standard deviation between the PMFs was then calculated.

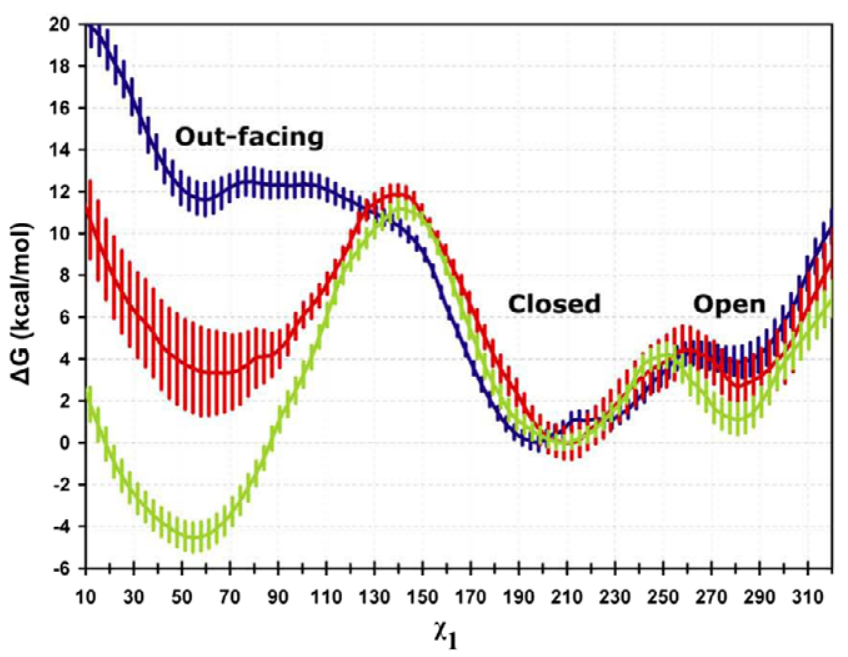

Figure 3. PMF for the $\chi_{1}$ rotation of N139 (blue), N139D (red) and N139D in the D132N/N139D double mutant (green).

\subsection{Continuum electrostatic calculations}

The $p \mathrm{~K}_{\mathrm{a}}$ shift calculations were performed with the PBEQ module of CHARMM [35]. Details pertaining to atomic radii used and dielectric constant values assigned to different regions of the system can be found in our previous study [32]. No explicit water molecules were included in the PBEQ calculation. The Poisson-Boltzmann (PB) equation was solved numerically by finite difference on a coarse grid $(0.60 \AA$ mesh size) focused on regions of interest up to a $0.30 \AA$ mesh size. The $p \mathrm{~K}_{\mathrm{a}}$ values are averaged over 150 snapshots selected randomly from five independent $2.5 \mathrm{~ns}$ molecular dynamics trajectories of the wild-type protein and both mutants. These calculations were performed separately for the closed and outfacing conformational states of residue N139D in the single or double mutants.

\section{Results and discussion}

\subsection{Conformational isomerization of residue 139}

The rotameric states of residue 139 in the wild-type, singlepoint mutant N139D, and double mutant D132N/N139D were characterized using free energy simulations combining umbrella sampling [38] and distributed replica sampling [39, 40, 42]. Figures 2 and 3 depict representative conformations of residue 139 in the wider context of the D channel and the PMF for the conformational isomerization of the $\chi_{1}$ torsion of residue 139 , respectively. Our previous study of functional hydration in the $\mathrm{D}$ channel characterized the conformational equilibrium between the 'closed' and 'open' conformations of $\mathrm{N} 139$, in which the side chain prevents and allows the formation of a hydrogen-bonded chain of water molecules, respectively (figures 2(a) and (b)) [11]. The conformational isomerization between the preferred closed state and the metastable open state was inferred to provide a conformational gate for proton uptake, since the presence of hydrogen bonds is a prerequisite for water-mediated proton relay [9] across the narrow bottleneck of the $\mathrm{D}$ channel. 
Here we have extended the range of the PMF to include rotation of the terminal group towards the entrance of the D channel, in the vestibule lying between residues 132 and 139 (figure 2(c)). This conformation will be referred to as the out-facing conformation from this point on. The free energy profiles reveal that, although the side chain of residue 139 in all three enzymes can adopt all three rotameric states (closed, open, out-facing), the stability of the out-facing conformer relative to the other two conformations varies dramatically (figure 3).

As we have shown previously [11], the closed state of residue N139 is favoured over the open state by approximately $4 \mathrm{kcal} \mathrm{mol}^{-1}$. Furthermore, the out-facing conformation is highly disfavoured in the wild-type enzyme, with a free energy of approximately $12 \mathrm{kcal} \mathrm{mol}^{-1}$ relative to the closed state. In the single-point mutant $\mathrm{N} 139 \mathrm{D}$, the equilibrium between open and closed states does not differ significantly from that of the wild-type, with a $3 \mathrm{kcal} \mathrm{mol}^{-1}$ preference for the closed state. However, the free energy of the out-facing conformer is now comparable to that of the open state. In contrast, in the N139D/D132N double mutant the out-facing conformer undergoes a dramatic stabilization relative to the single-point mutant and becomes preferred over closed and open states by 5 and $6 \mathrm{kcal} \mathrm{mol}^{-1}$, respectively. Based on these results, the most populated conformations of residue 139 (over 98\%) are predicted to be the closed, closed and out-facing states in the wild-type enzyme and in N139D and D132N/N139D mutants, respectively. These results justify, a posteriori, the neglect of the out-facing conformation in our previous studies of the wildtype and single-point mutant [32] while forcing us to consider its role in the restored phenotype of the double mutant.

The high energetic penalty incurred when residue N139 adopts the out-facing state is likely due to poor hydrogenbonding interactions between the amide terminus of N139 and chemical groups lining the vestibule of the D channel. In the single-point mutant $\mathrm{N} 139 \mathrm{D}$, the relative stabilization of the out-facing residue is likely to be due at least in part to the better hydration of the charged carboxylate group in the vestibule of the D channel, compared to the closed and open conformers of N139D (compare the hydrated state of the N139D side chain in figures 2(b) and (c)). However, the outfacing conformation also brings the two carboxylate groups of D132 and N139D in closer proximity to each other, increasing the Coulombic repulsion between these two groups. Indeed, we hypothesize that the dramatic stabilization of the out-facing conformation in the double mutant is due to the removal of the charge-charge repulsion resulting from the neutralization of residue 132. Residue 132, at the entrance of the channel, lies approximately $6 \AA$ from residue 139. As shown in our previous study of the single N139D mutant [32] and in the following section, electrostatic interactions between the charged groups of N139D and E286, which lie at least $18 \AA$ apart in the D channel, are strong enough to induce significant $p \mathrm{~K}_{\mathrm{a}}$ shifts.

If the charge state of residue 132 modulates the conformational equilibrium of N139D, it may be expected that, reciprocally, the conformational equilibrium of D132 depends on the charge and the conformational state of residue 139 . Specifically, both the introduction of a charge in the single

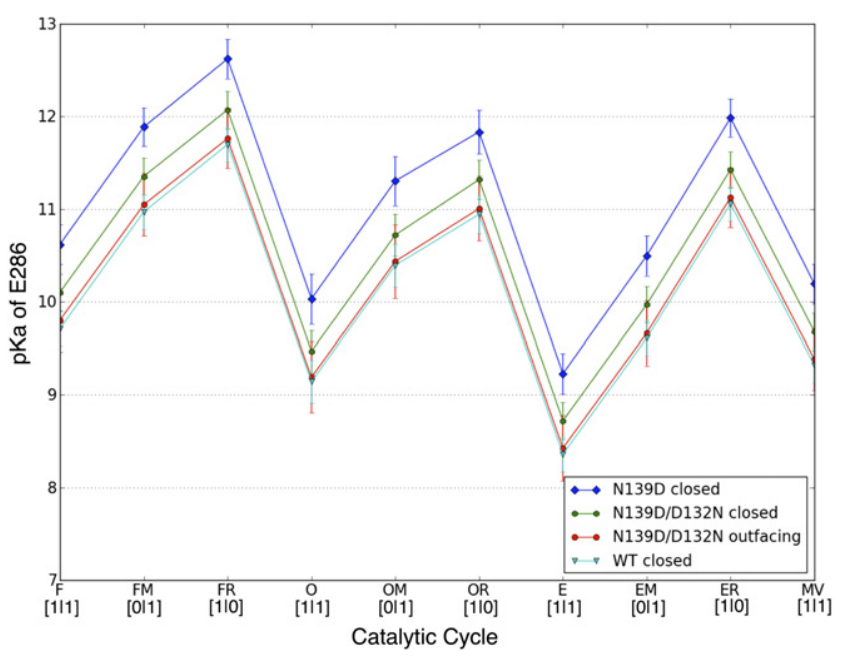

Figure 4. $p \mathrm{~K}_{\mathrm{a}}$ of E286 in successive catalytic steps in the wild-type enzyme (light blue), in the closed state of the single-point mutant N139D (dark blue) and of the double mutant D132N/N139D (green), and in the out-facing conformation of the double mutant (red). The letter codes refer to the catalytic states of the enzyme and the square brackets [0|1], [1|0] and [1|1] represent the distribution of charge in the two moieties of the active site, as defined in [32]. According to our electrostatic model, these three states correspond to proton delivery from E286 to the PLS, proton delivery from E286 to the $\mathrm{BNC}$, and proton pumping steps, respectively. The average $p \mathrm{~K}_{\mathrm{a}}$ of E286 in proton-delivery states [0|1] and [1|0] are 10.8 and 11.7 for the wild-type enzyme and the N139D single mutant, respectively. The lines connecting the $p \mathrm{~K}_{\mathrm{a}}$ values are shown to guide the eye. Standard deviations are within 0.3 units for all the calculated $p \mathrm{~K}_{\mathrm{a}}$ values.

N139D mutant and the conformational isomerization of that residue to its out-facing conformer may be expected to shift the conformational equilibrium of D132. However, the analysis of the rotameric states of the D132 side chain sampled in our simulation did not show any such dependence (results not shown). Resolving the issue of conformational coupling between residues 132 and 139 would necessitate a systematic characterization, using PMF calculations, of the rotameric equilibrium of residue D132 in different rotameric states of N139 and N139D. Such a study is beyond the scope of the present paper.

\subsection{Effect of mutations on the $p K_{a}$ of the proton shuttle E286}

In light of the above results, continuum electrostatic calculations were performed on the double mutant in the outfacing state and, for comparison, the closed state. Since the PMF profiles described above support the assumption that the closed states of N139 and N139D (single-point mutant) are the preferred conformers, calculations of the $p \mathrm{~K}_{\mathrm{a}}$ of E286 in the closed state of the wild-type and of the N139D single-point mutant are also included for comparison.

Figure 4 shows the calculated $p \mathrm{~K}_{\mathrm{a}}$ of E286 in successive catalytic steps of the enzyme. In this plot, [0|1], [1|0], and [1|1] denote the sequence of three alternating charge states of the active site of the enzyme, where the numbers on the left and on the right denote the total charge of heme a and the BNC, 
respectively [32]. Ten catalytic states denoted F through MV are shown, which are thought to involve the uptake of protons through the D channel, as described in detail in Fadda et al [32]. Together, these ten distinct catalytic states make up three out of four recurring subcycles in which electron and proton transfer are coupled electrostatically: the uptake of a proton by the PLS following reduction of heme a in state [0|1], the uptake of a chemical proton by the BNC following electron transfer from heme a to heme a3 in state [1|0], and the pumping of a proton upon the ensuing maximization of the positive charge in the active site of the enzyme (state [1|1]) [32]. As seen in figure 4 , the periodic recurrence of the three charge states induces a pseudo-periodic cycle in the $p \mathrm{~K}_{\mathrm{a}}$ of E286, the residue at the top of the $\mathrm{D}$ channel which shuttles protons from the D channel alternatively to the PLS and to the BNC in states $[0 \mid 1]$ and [1|0], respectively. In each of these cycles, the $p \mathrm{~K}_{\mathrm{a}}$ of E286 drops by up to two units in state [1|1].

As previously discussed [32], the introduction of a negatively charged residue at position 139 destabilizes the ionic state of residue E286 relative to its neutral, protonated state, resulting in a further increase in its already elevated proton affinity. This perturbation is significant in the closed conformer of N139D, which is the most populated state of the single mutant. However, this additional $p \mathrm{~K}_{\mathrm{a}}$ shift is smaller in the closed state of the double mutant and becomes negligible in the out-facing state, which is the predominant conformation of N139D in the double mutant (figure 4). The restoring effect of the double mutant, which brings the average $p \mathrm{~K}_{\mathrm{a}}$ of E286 within statistical uncertainty to that of the wild-type enzyme in the two proton-delivery states, is due to the increasing separation and better hydration of the carboxylate group of N139D in the out-facing state (see figure 2).

\subsection{Mechanism for the restoration of proton pumping activity}

The phenotype of the D132N/N139D double mutant is remarkable in its ability to restore wild-type activity to both the N139D decoupled mutant and the inactive D132N mutant [6]. As such, any proposed mechanism regarding the restored activity of the double mutant should include an explanation for both of these phenomena. Our results provide plausible explanations for both these experimental results.

When D132 is mutated to any non-carboxylate residue, the activity of the enzyme is reduced to $5 \%$ of wild-type [6] (see table 1). This is likely to be due to the removal of the ability for residue 132 to recruit protons to the entrance of the D channel, since the activity of the D132A mutant can be increased by increasing the concentration of protons on the matrix side of the protein [6]. Consequently, it is likely that the return to wild-type activity in the double mutant is due to the N139D carboxylate group acting as a replacement proton antenna. Our PMF results further support this hypothesis, since the low-energy out-facing rotamer of N139D in the double mutant places the carboxylate group of N139D a mere $6 \AA$ from the location of the wild-type D132 side chain in the crystallographic structure. In this orientation, it is inferred that residue N139D is sufficiently close to the matrix side of the protein as to act as a proton antenna. In addition, this outfacing rotamer is critical in explaining the phenotype of the double mutant in regards to the restoration of proton pumping activity to the N139D decoupled mutant.

In our previous study of decoupled single-point mutants N139D and N207D, we attributed the decoupling of proton pumping from redox chemistry to an increase in the $p \mathrm{~K}_{\mathrm{a}}$ of E286 small enough to allow proton delivery to the BNC but high enough to compromise proton delivery to the PLS [32]. Importantly, we also found that the magnitude of this $p \mathrm{~K}_{\mathrm{a}}$ shift was modulated by the proximity of the new charge to E286. Consistently with these findings, the above results show that swinging the N139D side chain to its out-facing conformation (figure 2), which is only marginally populated in the single mutant due to electrostatic repulsion with D132 but becomes the preferred conformer in the double mutant (figure 3) thanks to the neutralization of the charge of residue 132, brings the $p \mathrm{~K}_{\mathrm{a}}$ of E286 back to near-wild-type values (figure 4), consistent with the restoration of proton pumping [17].

\section{Conclusions}

We have examined the structural and electrostatic basis for decoupling and re-coupling of proton pumping and redox chemistry in the N139D and D132N/N139D mutants of CcO. The above results shed light onto the role of residues lining the D channel in the uptake and relay of protons to the active site of the enzyme. These findings are consistent with our previous analysis of structural fluctuations in the D channel [11] and support our previously proposed electrostatic model of proton pumping [32]. Detailed free energy simulations of the conformational equilibrium of residue 139 confirm that the closed conformer found in crystal structures of the enzyme is the lowest energy state in both the wild-type and the N139D mutant. In addition, this study has uncovered an out-facing conformer which is metastable in the wild-type protein and in the single N139D mutant but is predominant in the double mutant. Finally, electrostatic calculations suggest that the preference of the N139D carboxylate group for the out-facing conformation, where it may replace the 'proton antenna' lost in the neutralization of D132 while at the same time restoring the proton affinity of E286 and therefore its ability to deliver vectorial protons, restores proton pumping activity to the double mutant.

Thus, this study provides a consistent model explaining how the D132N/N139D double mutant re-establishes the electrostatic balance governing the uptake of protons in the enzyme, which was compromised both in D132N and N139D. Consistently with our proposed model of proton pumping, these findings underline the importance of electrostatic driving forces in the control of proton movement in the enzyme interior. In particular, our results suggest that preserving the spatial distribution of charges at the entrance of the D channel is necessary to guarantee both the uptake of protons and the subsequent relay of these protons to the catalytic site and the pumping element. More generally, our results also illustrate how energetic factors driving proton movement are modulated by conformational fluctuations of the enzyme. Although understanding the physical basis of proton uptake is an important step, much remains to be done to elucidate 
the molecular basis for the kinetic control necessary for preventing leakage and ensuring the directionality of proton movement in the pump cycle. In that perspective, further characterization of the interplay of conformational fluctuations and proton translocation is a challenge for future theoretical and computational work.

\section{Acknowledgments}

We thank the Centre for Computational Biology of the Hospital for Sick Children for a generous allocation of CPU resources and we gratefully acknowledge the Canadian Institutes of Health Research (grant MOP43949) for support. RP is a CRCP chairholder.

\section{References}

[1] Wikström M and Verkhovsky M I 2006 Towards the mechanism of proton pumping by the haem-copper oxidases Biochim. Biophys. Acta 1757 1047-51

[2] Brändén G, Gennis R B and Brzezinski P 2006 Transmembrane proton translocation by cytochrome c oxidase Biochim. Biophys. Acta 1757 1052-63

[3] Brzezinski P and Adelroth P 2006 Design principles of proton-pumping haem-copper oxidases Curr. Opin. Struct. Biol. 16 465-72

[4] Konstantinov A A et al 1997 The roles of the two proton input channels in cytochrome $\mathrm{c}$ oxidase from Rhodobacter sphaeroides probed by the effects of site-directed mutations on time-resolved electrogenic intraprotein proton transfer Proc. Natl Acad. Sci. USA 94 9085-90

[5] Brzezinski P and Adelroth P 1998 Pathways of proton transfer in cytochrome c oxidase J. Bioenerg. Biomembr. 30 99-107

[6] Mills D A et al 2000 Where is 'outside' in cytochrome c oxidase and how and when do protons get there? Biochim. Biophys. Acta 1458 180-7

[7] de Grotthuss C J T 1806 Mémoire sur la décompostion de l'eau et des corps qu'elle tient en dissolution à l'aide de l'électricité galvanique Ann. Chim. 58 54-74

[8] de Grotthuss C J T 2006 Memoir on the decomposition of water and of the bodies that it holds in solution by means of galvanic electricity Biochim. Biophys. Acta 1757 871-5

[9] Pomès R and Roux B 1996 Structure and dynamics of a proton wire: a theoretical study of $\mathrm{H}^{+}$translocation along the single-file water chain in the gramicidin channel Biophys. $J$. 71 19-39

[10] Svensson-Ek M et al 2002 The x-ray crystal structures of wild-type and EQ(I-286) mutant cytochrome C oxidases from rhodobacter sphaeroides J. Mol. Biol. 321 329-39

[11] Henry R et al 2009 Functional hydration and conformational gating of proton uptake in cytochrome c oxidase $\mathrm{J}$. Mol. Biol. 387 1165-85

[12] Xu J and Voth G A 2005 Computer simulation of explicit proton translocation in cytochrome c oxidase: the D-pathway Proc. Natl Acad. Sci. USA 102 6795-800

[13] Riistama S et al 1997 Bound water in the proton translocation mechanism of heam-copper oxidases FEBS Lett. 414 275-80

[14] Hofacker I and Schulten K 1998 Oxygen and proton pathways in cytochrome c oxidase Proteins Struct. Funct. Gen. 30 100-7

[15] Pomès R, Hummer G and Wikström M 1998 Structure and dynamics of a proton shuttle in cytochrome c oxidase Biochim. Biophys. Acta 1365 255-60

[16] Adelroth P et al 2000 Proton transfer from glutamate 286 determines the transition rates between oxygen intermediates in cytochrome c oxidase Biochim. Biophys. Acta 1459 533-9
[17] Brändén G et al 2006 Controlled uncoupling and recoupling of proton pumping in cytochrome c oxidase Proc. Natl Acad. Sci. USA 103 317-22

[18] Adelroth P et al 1997 Glutamate 286 in cytochrome aa3 from rhodobacter sphaeroides is involved in proton uptake during the reaction of the fully-reduced enzyme with dioxygen Biochemistry 36 13824-9

[19] Aagaard A et al 2000 Redesign of the proton-pumping machinery of cytochrome $\mathrm{c}$ oxidase: proton pumping does not require Glu(I-286) Biochemistry 39 15847-50

[20] Pawate A S et al 2002 A mutation in subunit I of cytochrome c oxidase from rhodobacter sphaeroides results in an increase in steady state activity but completely eliminates proton pumping Biochemistry 41 13417-23

[21] Mitchell D M et al 1996 Site-directed mutagenesis of residues lining a putative proton transfer pathway in cytochrome c oxidase from rhodobacter sphaeroides Biochemistry 35 13089-93

[22] Han D, Morgan J E and Gennis R B 2005 G204D, a mutation that blocks the proton-conducting D-channel of the aa3-type cytochrome c oxidase from rhodobacter sphaeroides Biochemistry 44 12767-74

[23] Han D et al 2006 Replacing Asn207 by aspartate at the neck of the $\mathrm{D}$ channel in the $\mathrm{aa}_{3}$-type cytochrome $\mathrm{c}$ oxidase from rhodobacter sphaeroides results in decoupling the proton pump Biochemistry 45 14064-74

[24] Backgren C et al 2000 Proton translocation by cytochrome c oxidase can take place without the conserved glutamic acid in subunit I Biochemistry 39 7863-7

[25] Pfitzner U et al 2000 Tracing the D-pathway in reconstituted site-directed mutants of cytochrome c oxidase from paracoccus denitrificans Biochemistry 39 6756-62

[26] Garcia-Horsman J A et al 1995 Proton transfer in cytochrome bo3 ubiquinol oxidase of Escherichia coli: second-site mutations in subunit I that restore proton pumping in the mutant Asp135 Biochemistry 34 4428-33

[27] Belevich I et al 2007 Exploring the proton pump mechanism of cytochrome c oxidase in real time Proc. Natl Acad. Sci. USA $1042685-90$

[28] Michel H 1998 The mechanism of proton pumping by cytochrome c oxidase Proc. Natl Acad. Sci. USA 95 12819-24

[29] Faxén K et al 2005 A mechanistic principle for proton pumping by cytochrome c oxidase Nature $437286-9$

[30] Belevich I, Verkhovsky M I and Wikström M 2006 Proton-coupled electron transfer drives the proton pump of cytochrome c oxidase Nature 440 829-32

[31] Wikström M and Verkhovsky M I 2007 Mechanism and energetics of proton translocation by the respiratory heme-copper oxidases Biochim. Biophys. Acta 1767 1200-14

[32] Fadda E, Yu C H and Pomès R 2008 Electrostatic control of proton pumping in cytochrome c oxidase Biochim. Biophys. Acta 1777 277-84

[33] MacKerell A D J et al 1998 All-atom empirical potential for molecular modeling and dynamics studies of proteins J. Phys. Chem. B 102 3586-616

[34] Jorgensen W L et al 1983 Comparison of simple potential functions for simulating liquid water J. Chem. Phys. 79 926-35

[35] Brooks B R et al 1983 CHARMM: a program for macromolecular energy minimization and dynamics calculations J. Comput. Chem. 4 187-217

[36] Ryckaert J P, Ciccotti G and Berendsen H J C 1977 Numerical-integration of cartesian equations of motion of a system with constraints - molecular-dynamics of $\mathrm{N}$-alkanes J. Comput. Phys. 23 327-41

[37] Humphrey W, Dalke A and Schulten K 1996 VMD: visual molecular dynamics J. Mol. Graph. 1433-8 
[38] Torrie G M and Valleau J P 1977 Nonphysical sampling distributions in Monte Carle free-energy estimation: umbrella sampling J. Comput. Phys. 23 187-99

[39] Rodinger T, Howell P L and Pomès R 2006 Distributed replica sampling J. Chem. Theory Comput. 2 725-31

[40] Neale C, Rodinger T and Pomès R 2008 Equilibrium exchange enhances the convergence rate of umbrella sampling Chem. Phys. Lett. $460375-81$

[41] Rodinger T, Howell P L and Pomès R 2008 Calculation of absolute protein-ligand binding free energy using distributed replica sampling J. Phys. Chem. 129155102

[42] Rauscher S, Neale C and Pomès R 2009 Simulated tempering distributed replica sampling, virtual replica exchange, and other generalized-ensemble methods for conformational sampling J. Chem. Theory Comput. 5 2640-62

[43] Kumar S et al 1995 Multidimensional free-energy calculations using the weighted histogram analysis method J. Comput. Chem. 16 1339-50

[44] Namslauer I et al 2010 A pathogenic mutation in cytochrome c oxidase results in impaired proton pumping while retaining $\mathrm{O}_{2}$-reduction activity Biochim. Biophys. Acta 1797 550-6
[45] Zhu J et al 2010 Decoupling mutations in the D-channel of the aa 3 -type cytochrome c oxidase from rhodobacter sphaeroides suggest that a continuous hydrogen-bonded chain of waters is essential for proton pumping Biochemistry 49 4476-82

[46] Fetter J R et al 1995 Possible proton relay pathways in cytochrome c oxidase Proc. Natl Acad. Sci. USA 92 1604-8

[47] Namslauer A et al 2003 Redox-coupled proton translocation in biological systems: proton shuttling in cytochrome c oxidase Proc. Natl Acad. Sci. USA 100 15543-7

[48] Dürr K L et al 2008 A D-pathway mutation decouples the Paracoccus denitrificans cytochrome c oxidase by altering the side-chain orientation of a distant conserved glutamate J. Mol. Biol. 384 865-77

[49] Lepp $\mathrm{H}$ et al 2008 Impaired proton pumping in cytochrome c oxidase upon structural alteration of the D pathway Biochim. Biophys. Acta 1777 897-903

[50] Lee H J et al 2010 Intricate role of water in proton transport through cytochrome c oxidase J. Am. Chem. Soc. 132 16225-39 